PROCEEDINGS OF THE

AMERICAN MATHEMATICAL SOCIETY

Volume 138, Number 9, September 2010, Pages 3203-3210

S 0002-9939(2010)10519-1

Article electronically published on May 14, 2010

\title{
SYMMETRY OF BOUND AND ANTIBOUND STATES IN THE SEMICLASSICAL LIMIT FOR A GENERAL CLASS OF POTENTIALS
}

\author{
SEMYON DYATLOV AND SUBHROSHEKHAR GHOSH
}

(Communicated by Hart F. Smith)

\begin{abstract}
We consider the Schrödinger operator $-h^{2} \partial_{x}^{2}+V(x)$ on a half-line, where $V$ is a compactly supported potential which is positive near the endpoint of its support. We prove that the eigenvalues and the purely imaginary resonances are symmetric up to an error $C e^{-\delta / h}$.
\end{abstract}

\section{INTRODUCTION}

In this paper, we study spectral properties of the Schrödinger operator

$$
P(h)=-h^{2} \partial_{x}^{2}+V(x)
$$

defined for $x$ in the half-line $(-\infty, B]$. Here $h>0$ is the semiclassical parameter and $V(x)$ is a piecewise continuous real-valued potential supported in $[0, B]$.

The operator $P(h)$ with the Neumann boundary condition at $B$ is self-adjoint on $L^{2}(-\infty, B)$; therefore, its resolvent

$$
R_{V}(\lambda)=\left(P(h)-\lambda^{2}\right)^{-1}, \operatorname{Im} \lambda>0,
$$

is a bounded operator from $L^{2}$ to $H^{2}$ for $\lambda^{2}$ not in the spectrum of $P(h)$. This resolvent can be extended meromorphically as an operator $L_{\text {comp }}^{2} \rightarrow H_{\text {loc }}^{2}$ to $\lambda \in \mathbb{C}$ with isolated poles of finite rank; these poles are called resonances. (The reader is referred to [12 for details.) To each resonance $\lambda$ corresponds a resonant state; that is, a nonzero $u \in H_{\text {loc }}^{2}(-\infty, B)$ solving the equation $\left(P(h)-\lambda^{2}\right) u=0$ with the Neumann boundary condition at the right endpoint and with the following outgoing condition at $-\infty$ :

$$
u(x)=A e^{-i \lambda x / h} \text { for all } x<0 \text { and some constant } A .
$$

(Note that for $x<0, u$ solves the free equation $\left(-h^{2} \partial_{x}^{2}-\lambda^{2}\right) u=0$, so it must be a linear combination of $e^{ \pm i \lambda x / h}$.)

For $\operatorname{Im} \lambda>0$, the outgoing condition implies that $u$ is exponentially decreasing on the negative half-line and thus $u \in L^{2}$; therefore, $\lambda$ is a pole (of the resolvent) lying in the upper half-plane if and only if $\lambda^{2}$ is an eigenvalue of $P(h)$ on $L^{2}$. Since $P(h)$ is self-adjoint, all poles in the upper half-plane have to lie on the imaginary axis. There may be poles $\lambda$ with $\operatorname{Im} \lambda<0$ and $\operatorname{Re} \lambda \neq 0$; however, we will restrict our attention to purely imaginary resonances:

Received by the editors December 2, 2009.

2010 Mathematics Subject Classification. Primary 34L25; Secondary 65L15, 81U20.

(C)2010 American Mathematical Society Reverts to public domain 28 years from publication 
Definition 1. A positive number $k$ is called a bound state if $i k$ is a pole of the resolvent $R_{V}(\lambda)$, and an antibound state if $-i k$ is a pole.

We see from above that $k$ is an (anti)bound state if and only if there exists a nonzero solution $u$ of the problem

$$
\begin{gathered}
\left(P(h)+k^{2}\right) u=0, \\
\left.u_{x}\right|_{x=B}=0, \\
h u_{x} \pm\left. k u\right|_{x=0}=0 .
\end{gathered}
$$

The plus sign in (3) corresponds to an antibound state, and the minus sign corresponds to a bound state. We will also study Neumann eigenvalues of $P(h)$ on $[0, B]$, i.e., those $k$ for which there exists a nonzero solution to (1) with boundary conditions (2) and

$$
\left.u_{x}\right|_{x=0}=0 .
$$

Since the space of solutions to (11) and (2) is always one dimensional, bound states, antibound states, and Neumann eigenvalues never coincide. However, Bindel and Zworski proved in [3] that bound and antibound states located away from zero coincide, modulo errors of order $e^{-\delta / h}$ for some $\delta>0$, if the potential satisfies the following conditions:

$$
\begin{gathered}
\exists A>0, V_{0}>0: V(x)=V_{0} \text { for all } x \in[0, A], \\
\exists \varepsilon>0: V(x)=0 \text { for all } x \in(A, A+\varepsilon) .
\end{gathered}
$$

In this paper, we prove a similar result with more general assumptions on the potential:

Theorem 1. Suppose that $V$ is a piecewise continuous real-valued potential supported in $[0, B]$ and satisfying the following bump condition:

$$
\exists A>0: V(x)>0 \text { for all } x \in(0, A] .
$$

Fix two constants $0<c_{k}<C_{k}<\infty$. Then there exist constants $C, \delta>0, h_{0}>0$ such that for $h<h_{0}$ and any $k \in\left[c_{k}, C_{k}\right]$ :

1. If $k$ is a Neumann eigenvalue, then there exist a bound state $k_{+}$and an antibound state $k_{-}$such that $\left|k-k_{ \pm}\right| \leq C e^{-\delta / h}$.

2. If $k$ is a bound or an antibound state, then there exists a Neumann eigenvalue $k_{0}$ such that $\left|k-k_{0}\right| \leq C e^{-\delta / h}$.

The bump condition (5) cannot be disposed of completely, as illustrated by the numerical experiments performed using [2]. Figure 1 shows two potentials on the whole line, each supported in $[-2,2]$, and the corresponding bound states (denoted by squares) and antibound states (denoted by circles). The vertical coordinate of each (anti)bound state on the picture corresponds to its value $k$; the horizontal coordinate corresponds to the value of $h^{-1}$ used. We see that the conclusion of the theorem does not appear to hold for the potential on the left, which does not satify the bump condition; at the same time, it is true for the potential on the right. Theorem 1, formulated for the half-line case, applies to these numerical experiments on the whole line since for even potentials, the set of their (anti)bound states is composed of these states for the positive half-line with Dirichlet condition and the same states for the Neumann condition; the theorem above can be applied with 

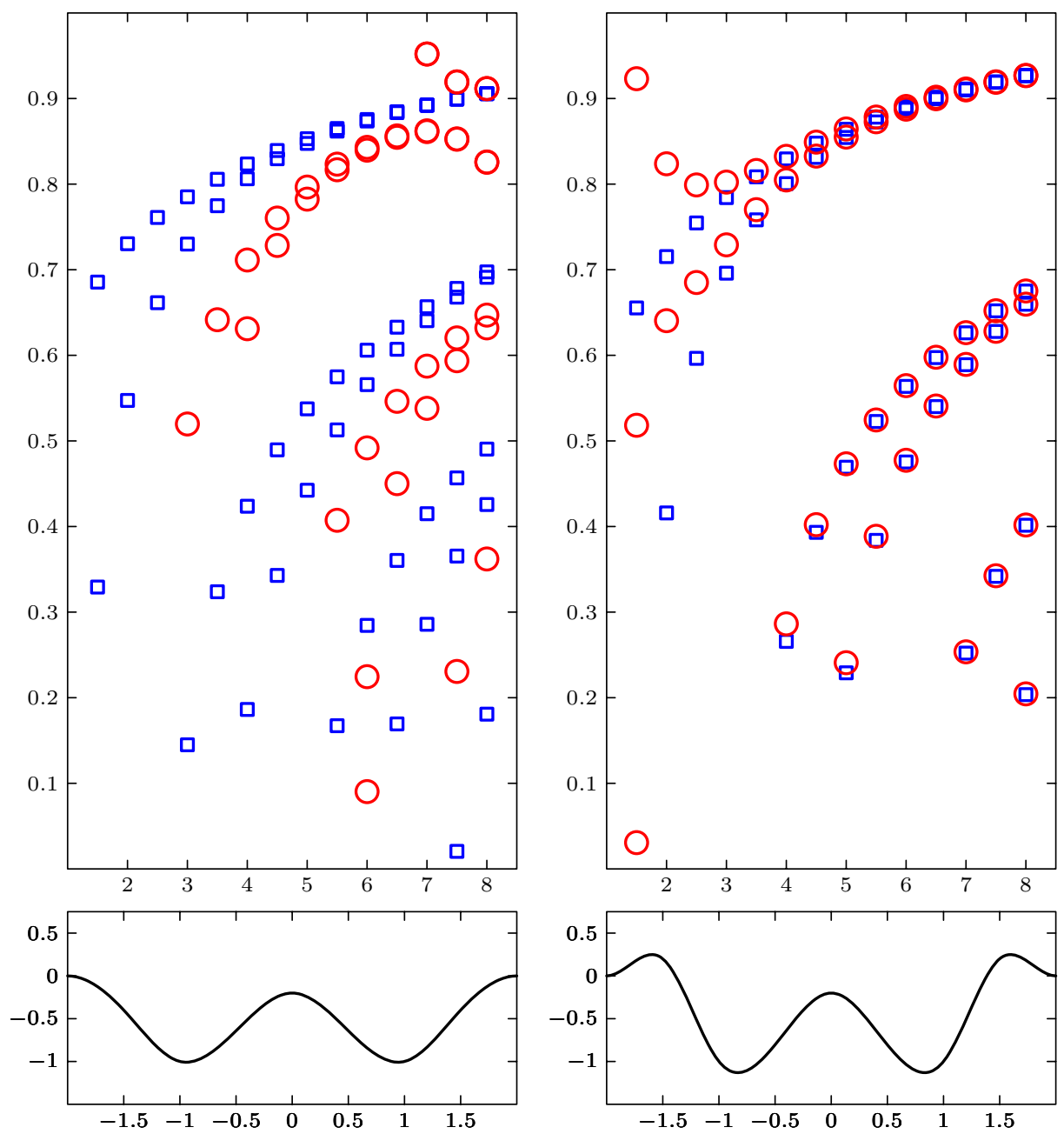

Figure 1. Bound and antibound states for two spline potentials (splinepot $([0,-0.4,-1,-0.2,-1,-0.4,0],[-2,-1.5$, $-1,0,1,1.5,2])$ and splinepot $([0,0.2,-1,-0.2,-1$, $0.2,0],[-2,1.5,-1,0,1,1.5,2]))$

Dirichlet condition in place of (2). (However, condition (4) cannot be replaced by the Dirichlet condition in the theorem.)

The study of resonances in one dimension has a long tradition going back to the origins of quantum mechanics; see for instance [8]. One of the first studies of their distribution was conducted by Regge [10]; since then, there have been many mathematical results on the topic, including [1, [4, [5], 6], 7], 9], 11, and [13. Concerning antibound states, Hitrik has shown in [6] that for a positive compactly supported potential, there are no antibound states in the semiclassical limit. This agrees with our result since there are no bound states in this case. Simon proved in [11 that between any two bound states, there must be an odd 
number of antibound states; the following corollary of this result follows almost immediately using the methods we develop to prove Theorem 1:

Theorem 2. Consider the half-line problem with a bounded compactly supported potential $V$ (which does not need to satisfy any positivity condition). Then for each two bound states $0<k_{1}<k_{2}$, the interval $\left(k_{1}, k_{2}\right)$ contains at least one antibound state. In particular, if there are $n$ bound states in some subinterval of $(0, \infty)$, then there are at least $n-1$ antibound states in the same subinterval.

The proof of Theorem 1 works as follows: we study the evolution (in $x$ ) of the vectors $\left(u, h u_{x}\right)$ for the three solutions of (1D) with initial data at $x=0$ satisfying the conditions (3) and (4). The idea is to look at these three vectors at $x=A$. Since $V(x)+k^{2} \geq 0$ on the interval $(0, A)$, the transition matrix for the considered vectors from $x=0$ to $x=A$ will have an expanding and a contracting direction. (In fact, if we introduce rescaling $\tilde{x}=x / h$, then the behavior of the original system for small $h$ is similar to the behavior of the rescaled system for large $\tilde{x}$, and the latter will be similar to the behavior of the geodesic flow on a two-dimensional manifold of negative curvature.) It turns out that our three vectors lie in a certain angle between the expanding and the contracting directions, from which it follows that they will stay in this angle for later times (Lemma 2); what is more, their polar angles will get exponentially close to each other (Lemma 7). Finally, we can study how the polar angles of the considered vectors change with $k$ (Lemma 4): it follows (Lemma 8) that the polar angle for the solution with Neumann initial data at $x=0$ will strictly increase in $k$ and the polar angle for the solution with the same data at $x=B$ will decrease in $k$. The proof is then completed by a pertrubation argument (Lemma 5).

The detailed proofs of Theorems 1 and 2 are given in Section 3. Both are elementary and use certain properties of ordinary differential equations presented in Section 2.

The authors would like to thank Maciej Zworski for suggesting the problem and for many illuminating discussions.

\section{Preliminaries}

Throughout this section, $I$ is an interval in $\mathbb{R}, V(x) \in L^{\infty}(I ; \mathbb{R}), u(x), v(x) \in$ $H^{2}(I ; \mathbb{R}), h>0$, and $P(h)=-h^{2} \partial_{x}^{2}+V(x)$. Any solution to the equation $P(h) u=0$ is determined by the vector $\left(u, h u_{x}\right)$ at any $x$; we will sometimes view this vector in polar coordinates:

Definition 2. Define the length $L(u)$ and the polar angle $\theta(u)$ by the equations

$$
\begin{gathered}
u=L(u) \cos \theta(u), \\
h u_{x}=L(u) \sin \theta(u) .
\end{gathered}
$$

Here $\theta(u)$ lies in the circle $\mathbb{S}^{1}=\mathbb{R} /(2 \pi \mathbb{Z})$.

Lemma 1. Define the Wronskian $W(u, v)$ by

$$
W(u, v)=h\left(u v_{x}-v u_{x}\right) .
$$

Then

$$
\begin{aligned}
& W(u, v)=L(u) L(v) \sin (\theta(v)-\theta(u)), \\
& h \partial_{x} W(u, v)=v \cdot P(h) u-u \cdot P(h) v .
\end{aligned}
$$


Note that $W(u, v)$ is just the oriented area of the parallelogram spanned by the vectors $\left(u, h u_{x}\right)$ and $\left(v, h v_{x}\right)$. The next lemma tells us that if the vector $\left(u, h u_{x}\right)$ falls inside a certain angle in the plane at the initial time, then it will stay inside that angle for all later times:

Lemma 2. Suppose that $a^{2} \leq V(x) \leq b^{2}$ for all $x \in I$ and some constants $a, b>0$. Let $u$ be a solution to $P(h) u=0$ and define

$$
W_{+}(u)=W\left(u, e^{b x / h}\right), W_{-}(u)=W\left(e^{-a x / h}, u\right) .
$$

Let $x_{0}$ be a point in $I$ and assume that $W_{+}(u), W_{-}(u) \geq 0$ at $x_{0}$. Then for $x \geq x_{0}$, the functions $W_{ \pm}(u)$ are increasing in $x$ and

$$
u \geq \frac{L(u)}{\sqrt{1+b^{2}}}
$$

Proof. We have

$$
e^{-b x / h} W_{+}(u)=b u-h u_{x}, e^{a x / h} W_{-}(u)=a u+h u_{x} .
$$

Therefore, $W_{+}(u), W_{-}(u) \geq 0$ yields $\left|h u_{x}\right| \leq b u$ and thus (8). Next,

$$
\begin{aligned}
P(h) e^{b x / h} & =e^{b x / h}\left(V(x)-b^{2}\right) \leq 0, \\
P(h) e^{-a x / h} & =e^{-a x / h}\left(V(x)-a^{2}\right) \geq 0 .
\end{aligned}
$$

Using (7), we see that $\partial_{x} W_{ \pm} \geq 0$ as long as $u \geq 0$. It remains to prove that $u(x) \geq 0$ for $x \geq x_{0}$. Suppose this is false and let $x_{1}=\inf \left\{x \geq x_{0} \mid u(x)<0\right\}$. Then $u$ is not identically zero; since it solves a second order linear ODE, $L(u)>0$ everywhere. But $u \geq 0$ on $\left[x_{0}, x_{1}\right]$, so $W_{ \pm}$are increasing on this interval. In particular, $W_{ \pm} \geq 0$ at $x_{1}$ and thus (8) holds at this point. However, by the choice of $x_{1}$ we have $u\left(x_{1}\right)=0$, which contradicts $L(u)>0$.

In the next section, we will use the following crude estimate on how fast the solutions of an ODE can grow:

Lemma 3. Assume that $|V(x)| \leq M$ for $x \in I$ and that $u$ is a solution to $P(h) u=$ 0 . Take $x_{0}, x_{1} \in I$; then

$$
\left.L(u)\right|_{x=x_{1}} \leq\left. e^{(1+M)\left|x_{0}-x_{1}\right| /(2 h)} \cdot L(u)\right|_{x=x_{0}} .
$$

Proof. Without loss of generality we may assume that $x_{1}>x_{0}$. We have $L(u)^{2}=$ $u^{2}+\left(h u_{x}\right)^{2} ;$ thus

$$
h \partial_{x}\left(L(u)^{2}\right)=2 h u u_{x}(1+V(x)) \leq(1+M) L(u)^{2},
$$

and the lemma is proven by Gronwall's inequality.

Lemma 4. Assume that $u(x, k)$ is a family of solutions to $\left(P(h)+k^{2}\right) u=0$, $x_{0}, x_{1} \in I$, and $u\left(x_{0}, k\right)$ and $u_{x}\left(x_{0}, k\right)$ are independent of $k$. Let $\Theta_{1}(k)=$ $\left.\theta(u(x, k))\right|_{x=x_{1}}, L_{1}(k)=\left.L(u(x, k))\right|_{x=x_{1}}$. Then

$$
\Theta_{1}^{\prime}(k)=\frac{2 k}{h L_{1}(k)^{2}} \int_{x_{0}}^{x_{1}} u(x, k)^{2} d x .
$$

Proof. We have $\left.W\left(u, u_{k}\right)\right|_{x=x_{1}}=L_{1}(k)^{2} \Theta_{1}^{\prime}$. (To see that, differentiate the formulas in Definition 2 in $k$ and use the definition of the Wronskian.) Now, we differentiate the equation $\left(P(h)+k^{2}\right) u=0$ in $k$ to get $\left(P(h)+k^{2}\right) u_{k}=-2 k u$. It remains to apply (7) together with $\left.W\left(u, u_{k}\right)\right|_{x=x_{0}}=0$. 
Lemma 5. Assume that $\Phi$ is a $C^{1}$ map from the interval $I=\left[K_{0}, K_{1}\right]$ to the circle $S^{1}=\mathbb{R} / 2 \pi \mathbb{Z}$ and $\Phi^{\prime}(k) \geq \delta>0$ for all $k \in I$. Suppose that $\Psi: I \rightarrow \mathbb{S}^{1}$ is a continuous map such that $|\Psi(k)| \leq \varepsilon<\pi$ for all $k$. Put $\nu=\varepsilon / \delta$ and $I_{\nu}=$ $\left[K_{0}+\nu, K_{1}-\nu\right]$. Then:

1. If $k_{0} \in I_{\nu}$ has $\Phi\left(k_{0}\right)=0$, then there exists $k_{1} \in I$ with $\Phi\left(k_{1}\right)=\Psi\left(k_{1}\right)$ and $\left|k_{0}-k_{1}\right| \leq \nu$.

2. If $k_{1} \in I_{\nu}$ has $\Phi\left(k_{1}\right)=\Psi\left(k_{1}\right)$, then there exists $k_{0} \in I$ with $\Phi\left(k_{0}\right)=0$ and $\left|k_{0}-k_{1}\right| \leq \nu$.

Proof. We can lift $\Phi$ and $\Psi$ to continuous maps $\bar{\Phi}, \bar{\Psi}: I \rightarrow \mathbb{R}$; then $|\bar{\Psi}| \leq \varepsilon$ and $\bar{\Phi}\left(k^{\prime}\right)-\bar{\Phi}(k) \geq \delta\left(k^{\prime}-k\right)$ for $k^{\prime} \geq k$.

1. We have $\bar{\Phi}\left(k_{0}\right)=2 \pi m$ for some $m \in \mathbb{Z}$. Then $\bar{\Phi}\left(k_{0}+\nu\right) \geq 2 \pi m+\delta \nu \geq$ $2 \pi m+\bar{\Psi}\left(k_{0}+\nu\right)$ and $\bar{\Phi}\left(k_{0}-\nu\right) \leq 2 \pi m+\bar{\Psi}\left(k_{0}-\nu\right)$; it remains to apply the intermediate value theorem.

2. Similar to the previous statement, we have $\bar{\Phi}\left(k_{1}\right)=2 \pi m+\bar{\Psi}\left(k_{1}\right)$ for some $m \in \mathbb{Z}$ and $\bar{\Phi}\left(k_{1}+\nu\right) \geq 2 \pi m \geq \bar{\Phi}\left(k_{1}-\nu\right)$.

Lemma 6. Assume that $\Phi$ is a $C^{1}$ map from some interval $I$ to the circle $\mathbb{S}^{1}=$ $\mathbb{R} /(2 \pi \mathbb{Z})$ with $\Phi^{\prime}(k)>0$ for all $k \in I$. Let $\Psi: I \rightarrow \mathbb{S}^{1}$ be a continuous map such that $\Psi(k) \neq 0$ for all $k \in I$. If $k_{1}<k_{2}$ are two roots of the equation $\Phi=0$, then the interval $\left(k_{1}, k_{2}\right)$ contains at least one root of the equation $\Phi=\Psi$.

Proof. As in the previous lemma, lift $\Phi$ and $\Psi$ to maps $\bar{\Phi}, \bar{\Psi}: I \rightarrow \mathbb{R}$; we can make $0<\bar{\Psi}(k)<2 \pi$ for all $k \in I$. Since $\bar{\Phi}^{\prime}>0$ everywhere, we have $\bar{\Phi}\left(k_{j}\right)=2 \pi m_{j}$, where $m_{1}<m_{2}$ are some integers. Therefore, $\bar{\Phi}\left(k_{1}\right)<2 \pi m_{1}+\bar{\Psi}\left(k_{1}\right)$ and $\bar{\Phi}\left(k_{2}\right)>$ $2 \pi m_{1}+\bar{\Psi}\left(k_{2}\right)$; it remains to apply the intermediate value theorem.

\section{Proofs of the theorems}

We assume in this section that $0<c_{k}^{\prime} \leq k \leq C_{k}^{\prime}$ for some constants $c_{k}^{\prime}<c_{k}$ and $C_{k}^{\prime}>C_{k}$; the constants in our estimates will depend on $c_{k}^{\prime}$ and $C_{k}^{\prime}$. (We need to expand the interval $\left[c_{k}, C_{k}\right]$ a little bit to be able to apply Lemma 5 , )

Consider the solutions $u_{ \pm}, u_{0}, u_{1}(x, k)$ to the equation (1) in $[0, B]$ with the initial data

$$
\begin{gathered}
u_{ \pm 0}(0, k)=1, \partial_{x} u_{0}(0, k)=0, h \partial_{x} u_{ \pm}(0, k)= \pm k, \\
u_{1}(B, k)=1, \partial_{x} u_{1}(B, k)=0 .
\end{gathered}
$$

Define $\Theta_{0}(k), \Theta_{ \pm}(k)$, and $\Theta_{1}(k)$ to be the polar angles of vectors $\left(u, h u_{x}\right)$ at $x=A$ for $u=u_{0}, u_{ \pm}, u_{1}$. Then $k>0$ is

- a Neumann eigenvalue if $u_{0}$ and $u_{1}$ are linearly dependent; that is (recalling that they solve the same second order ODE), if $2\left(\Theta_{0}(k)-\Theta_{1}(k)\right)=0$;

- a bound state if $2\left(\Theta_{+}(k)-\Theta_{1}(k)\right)=0$;

- an antibound state if $2\left(\Theta_{-}(k)-\Theta_{1}(k)\right)=0$.

Here we count angles modulo $2 \pi$.

To prove Theorem 1, it suffices to use Lemma 5 (for $\Phi=2\left(\Theta_{0}-\Theta_{1}\right.$ ) and $\left.\Psi=2\left(\Theta_{0}-\Theta_{ \pm}\right)\right)$together with the following two facts:

Lemma 7. For some constants $C_{1}$ and $\delta_{1}>0$ independent of $h$ and $k$,

$$
\left|2\left(\Theta_{0}(k)-\Theta_{ \pm}(k)\right)\right| \leq C_{1} e^{-\delta_{1} / h} \text { for all } k \in\left[c_{k}^{\prime}, C_{k}^{\prime}\right] .
$$

Lemma 8. We have $\Theta_{0}^{\prime}(k)-\Theta_{1}^{\prime}(k) \geq 1 / C_{2}>0$ for all $k \in\left[c_{k}^{\prime}, C_{k}^{\prime}\right]$ and some constant $C_{2}$ independent of $h$ and $k$. 
We first prove Lemma 7. Put $b=\max _{[0, A]} V(x), k_{b}=\sqrt{k^{2}+b}, \psi_{0}(x)=e^{-k x / h}$, $\psi_{+}(x)=e^{k_{b} x / h}$, and consider the Wronskians

$$
W_{+}(u)=W\left(u, \psi_{+}\right), W_{0}(u)=W\left(\psi_{0}, u\right) .
$$

These are nonnegative for $u=u_{0}, u_{ \pm}$at $x=0$. Then by Lemma 2, all these six functions are nonnegative and increasing in $x$ for $0 \leq x \leq A$.

Our first goal is to get an exponential lower bound on the length $L(u)$ for $u=$ $u_{0}, u_{ \pm}$at $x=A$. For $u_{0}$, note that by (6)

$$
L\left(u_{0}\right) \geq \frac{W\left(\psi_{0}, u_{0}\right)}{L\left(\psi_{0}\right)} \geq \frac{\left.W_{0}\left(u_{0}\right)\right|_{x=0}}{L\left(\psi_{0}\right)} \geq \frac{1}{C} e^{k x / h} .
$$

The same applies to $u_{+}$. However, $u_{-}$needs more careful analysis since $W_{0}\left(u_{-}\right)=0$ at $x=0$. For that, take $0<t<1$ and put $a=\min _{[t A, A]} V(x)>0, k_{a}=\sqrt{k^{2}+a}$, $\psi_{-}(x)=e^{-k_{a} x / h}$, and $W_{-}(u)=W\left(\psi_{-}, u\right)$. First, we have by Lemma 3

$$
L\left(u_{-}\right) \geq\left. e^{-\left(1+k^{2}+b\right) x /(2 h)} \cdot L\left(u_{-}\right)\right|_{x=0} .
$$

Next, $W_{0}\left(u_{-}\right) \geq 0$ and $W_{+}\left(u_{-}\right) \geq 0$, so by (8)

$$
W_{-}\left(u_{-}\right) \geq\left(k_{a}-k\right) u_{-} \psi_{-} \geq \frac{1}{C} L\left(u_{-}\right) \psi_{-} .
$$

Finally, we apply Lemma 2 on the interval $[t A, A]$ to get

$$
\left.L\left(u_{-}\right)\right|_{x=A} \geq \frac{\left.W_{-}\left(u_{-}\right)\right|_{x=t A}}{\left.L\left(\psi_{-}\right)\right|_{x=A}} \geq \frac{1}{C} e^{\left(k(1-t)-\left(1+k^{2}+b\right) t\right) A / h} .
$$

For $t$ small enough and all $k, k(1-t)-\left(1+k^{2}+b\right) t \geq 0$, so we have

$$
\left.L\left(u_{-}\right)\right|_{x=A} \geq \frac{1}{C}>0 .
$$

The next step is to use that $u_{0}$ and $u_{ \pm}$solve the same equation (1) and thus $W\left(u_{0}, u_{ \pm}\right)$is constant in $x$. Therefore, at $x=A$ we have by (6)

$$
\left|\sin \left(\theta\left(u_{ \pm}\right)-\theta\left(u_{0}\right)\right)\right|=\frac{\left|W\left(u_{0}, u_{ \pm}\right)\right|}{L\left(u_{0}\right) L\left(u_{ \pm}\right)} \leq C e^{-k A / h} .
$$

That finishes the proof of Lemma 7 .

To prove Lemma 8, first note that by Lemma 4, $\Theta_{1}^{\prime}(k) \leq 0$ and

$$
\Theta_{0}^{\prime}(k) \geq \frac{1}{\left.C h L\left(u_{0}\right)^{2}\right|_{x=A}} \int_{0}^{A}\left|u_{0}(x, k)\right|^{2} d x .
$$

By (8), $u_{0} \geq L\left(u_{0}\right) / C$. Also, by Lemma $3, L\left(u_{0}\right) \geq\left. e^{C(x-A) / h} L\left(u_{0}\right)\right|_{x=A}$ for $0 \leq$ $x \leq A$; thus

$$
\int_{0}^{A}\left|u_{0}(x, k)\right|^{2} d x \geq \frac{1}{C} \int_{0}^{A} e^{C(x-A) / h}\left(\left.L\left(u_{0}\right)^{2}\right|_{x=A}\right) d x \geq\left.\frac{h}{C} L\left(u_{0}\right)^{2}\right|_{x=A}
$$

and Lemma 8 is proven, which finishes the proof of Theorem 1.

To prove Theorem 2, let $\Phi_{ \pm}(k)=\left.\theta\left(u_{ \pm}\right)\right|_{x=B}$; a bound state corresponds to $2 \Phi_{+}=0$ and an antibound state corresponds to $2 \Phi_{-}=0$. Since $\left.\theta\left(u_{+}\right)\right|_{x=0}$ is increasing with $k$, by an argument similar to the proof of Lemma 4 we get $\Phi_{+}^{\prime}(k)>0$ for all $k$. Moreover, $2\left(\Phi_{+}(k)-\Phi_{-}(k)\right)$ is never zero, as this would correspond to $u_{+}$and $u_{-}$being linearly dependent. We may now apply Lemma 6 with $\Phi=2 \Phi_{+}$ and $\Psi=2\left(\Phi_{+}-\Phi_{-}\right)$. 


\section{REFERENCES}

[1] A. A. Abramov, A. Aslanyan, and E. B. Davies, Bounds on complex eigenvalues and resonances, J. Phys. A. 34(2001), 57-72. MR1819914 (2002c:81225)

[2] D. Bindel, MatScat: MATLAB Codes for 1D Potential Scattering, http://cims.nyu.edu/ dbindel/matscat.

[3] D. Bindel and M. Zworski, Symmetry of bound and antibound states in the semiclassical limit, Lett. Math. Phys. 81(2007), 107-117. MR2336226 (2008f:81274)

[4] P. Briet, J.-M. Combes, and P. Duclos, On the location of resonances for Schrödinger operators in the semi-classical limit. II, Comm. Partial Differential Equations 12(1987), 201-222. MR 876987(89e:81028b)

[5] R. Froese, Asymptotic distribution of resonances in one dimension, J. of Differential Equations 137(2) (1997), 251-272. MR1456597 (98f:81339)

[6] M. Hitrik, Bounds on scattering poles in one dimension, Comm. Math. Phys. 208(1999), 381-411. MR1729092 (2000m:34192)

[7] E. Korotyaev, Inverse resonance scattering on the real line, Inverse Problems 21(2005), 325341. MR2146179 (2006a:34033)

[8] L. D. Landau and E. M. Lifshitz, Quantum Mechanics: Non-Relativistic Theory, Third Edition, Elsevier, 1977. MR0093319 (19:1230k)

[9] L. Nedelec, Asymptotics of resonances for a Schrödinger operator with matrix values, math.SP/0509391.

[10] T. Regge, Analytic properties of the scattering matrix, Nuovo Cimento 10(1958), 671-679. MR0095702 (20:2203)

[11] B. Simon, Resonances in one dimension and Fredholm determinants, J. Funct. Anal. 178(2000), 396-420. MR1802901 (2001j:34031)

[12] S.-H. Tang and M. Zworski, Potential Scattering on the Real Line, lecture notes, http: //math.berkeley.edu/ zworski/tz1.pdf.

[13] M. Zworski, Distribution of poles for scattering on the real line, J. Funct. Anal. 73(1987), 277-296. MR899652(88h:81223)

Department of Mathematics, Evans Hall, University of California, Berkeley, CaliFORNIA 94720

E-mail address: dyatlov@math.berkeley.edu

Department of Mathematics, Evans Hall, University of California, Berkeley, CaliFORNIA 94720

E-mail address: subhro@math.berkeley.edu 